\title{
ARTICLE OPEN \\ Newcastle disease virus-based H5 influenza vaccine protects chickens from lethal challenge with a highly pathogenic H5N2 avian influenza virus
}

\author{
Jingjiao Ma ${ }^{1,6}$, Jinhwa Lee ${ }^{1}$, Haixia Liu ${ }^{1}$, Ignacio Mena ${ }^{2,3}$, A. Sally Davis ${ }^{1}$, Sun Young Sunwoo ${ }^{1}$, Yuekun Lang ${ }^{1}$, Michael Duff ${ }^{1}$, \\ Igor Morozov ${ }^{1}$, Yuhao $\mathrm{Li}^{1}$, Jianmei Yang ${ }^{1,4}$, Adolfo García-Sastre ${ }^{2,3,5}$, Juergen A. Richt ${ }^{1}$ and Wenjun Ma ${ }^{1}$
}

Since December 2014, Eurasian-origin, highly pathogenic avian influenza H5 viruses including H5N1, H5N2, and H5N8 subtypes (called $\mathrm{H} 5 \mathrm{~N} x$ viruses), which belong to the H5 clade 2.3.4.4, have been detected in U.S. wild birds. Subsequently, highly pathogenic H5N2 and H5N8 viruses have caused outbreaks in U.S. domestic poultry. Vaccination is one of the most effective ways to control influenza outbreaks and protect animal and public health. Newcastle disease virus (NDV)-based influenza vaccines have been demonstrated to be efficacious and safe in poultry. Herein, we developed an NDV-based H5 vaccine (NDV-H5) that expresses a codon-optimized ectodomain of the hemagglutinin from the A/chicken/lowa/04-20/2015 (H5N2) virus and evaluated its efficacy in chickens. Results showed that both live and inactivated NDV-H5 vaccines induced hemagglutinin inhibition antibody titers against the H5N2 virus in immunized chickens after prime and booster, and both NDV-H5 vaccines completely protected chickens from lethal challenge with the highly pathogenic H5N2 A/turkey/Minnesota/9845-4/2015 virus. No clinical signs and only minimal virus shedding was observed in both vaccinated groups. In contrast, all mock-vaccinated, H5N2-infected chickens shed virus and died within 5 days post challenge. Furthermore, one dose of the live NDV-H5 vaccine also provided protection of $90 \%$ chickens immunized by coarse spraying; after exposure to H5N2 challenge, sera from vaccinated surviving chickens neutralized both highly pathogenic H5N1 and H5N8 viruses. Taken together, our results suggest that the NDV-based H5 vaccine is able to protect chickens against intercontinental highly pathogenic $\mathrm{H} 5 \mathrm{~N} x$ viruses and can be used by mass application to protect the poultry industry.

npj Vaccines (2017)2:33; doi:10.1038/s41541-017-0034-4

\section{INTRODUCTION}

Highly pathogenic avian influenza (HPAl) H5N8 viruses have spread globally to many countries; firstly, they were reported in Korea, subsequently in 2014 in China, Japan, Germany, United Kingdom, and Italy. ${ }^{1,2}$ A reassortant HPAI H5N2 virus that contains 5 gene segments (PB2, PA, HA, M, and NS) related to Eurasian HPAI H5N8 was found in Canada in November 2014. ${ }^{3}$ The US Department of Agriculture (USDA) confirmed HPAI H5N8 and H5N2 virus infections in wild birds in the state of Washington in December, 2014. ${ }^{4}$ The H5N8, and reassortant H5N2, and H5N1 viruses (altogether called $\mathrm{H} 5 \mathrm{Nx}$ viruses), in which the $\mathrm{H} 5$ gene belongs to clade 2.3.4.4, have been subsequently detected in $21 \mathrm{U}$. S. States, where 15 States reported outbreaks in domestic poultry and 6 States only in wild birds. ${ }^{5}$ The control of the 2015 HPAl outbreaks in the U.S. required euthanasia of approximately 49 million domestic birds (42 million chickens, 7 million turkeys) according to USDA reports. ${ }^{6}$ Both $\mathrm{H} 5 \mathrm{~N} 2$ and $\mathrm{H} 5 \mathrm{~N} 1$ viruses found in the U.S. are reassortant viruses, the latter one has only been found in the wild birds. The H5N2 virus contains PB2, PA, HA, M, and NS genes from the Eurasian HPAI H5N8 virus and the PB1, NP, and NA genes from North American low pathogenic avian influenza viruses, ${ }^{7}$ while the $\mathrm{H} 5 \mathrm{~N} 1$ virus contains $\mathrm{PB} 2, \mathrm{HA}, \mathrm{NP}$, and $\mathrm{M}$ genes from the Eurasian $\mathrm{H} 5 \mathrm{~N} 8$ virus and the PB1, PA, NA, and NS genes from North American low pathogenic avian influenza viruses. ${ }^{8}$ The $\mathrm{HA}$ genes of both HPAI H5N1 and H5N2 viruses are genetically related to the $\mathrm{H} 5 \mathrm{~N} 8$ virus earlier isolated in South Korea. The homology of $\mathrm{H} 5$ genes among HPAI H5N1, H5N2, and H5N8 viruses are more than $99 \%$ at the nucleotide level. ${ }^{7}$ Intercontinental $\mathrm{H} 5 \mathrm{Nx}$ viruses can infect wild birds without clinical signs but cause severe disease and death in domestic birds. The introduction of the HPAI $\mathrm{H} 5 \mathrm{~N} x$ viruses into U.S. domestic poultry in 2015 has resulted in significant economic losses for the poultry industry (estimated to be $\$ 4$ billion in the U.S.) and international trade issues.

Although the culling or stamp out of infected poultry is an effective way to prevent the spread of HPAl viruses in isolated outbreaks, these efforts are compromised by violating poultry movement control and surveillance around outbreaks. In addition, there is a limited knowledge and capacity for safe and humane culling of millions of poultry and disposal. As the incidence of outbreaks within a country increases, animal health authorities can rapidly become overwhelmed through lack of resources and

\footnotetext{
${ }^{1}$ Department of Diagnostic Medicine/Pathobiology, College of Veterinary Medicine, Kansas State University, Manhattan, KS, USA; ${ }^{2}$ Department of Microbiology, Icahn School of Medicine at Mount Sinai, New York, NY, USA; ${ }^{3}$ Global Health and Emerging Pathogens Institute, Icahn School of Medicine at Mount Sinai, New York, NY, USA; ${ }^{4}$ Innovation Team for Pathogen Ecology Research on Animal Influenza Virus, Department of Avian Infectious Disease, Shanghai Veterinary Research Institute, Chinese Academy of Agricultural Sciences, Shanghai, China and ${ }^{5}$ Department of Medicine, Division of Infectious Diseases, Icahn School of Medicine at Mount Sinai, New York, NY, USA Correspondence: Adolfo García-Sastre (Adolfo.Garcia-Sastre@mssm.edu) or Juergen A. Richt (jricht@vet.k-state.edu) or Wenjun Ma (wma@vet.k-state.edu) ${ }^{6}$ Present address: Shanghai Jiao Tong University, Shanghai, China Jingjiao Ma and Jinhwa Lee contributed equally to this work.
}

Received: 5 June 2017 Revised: 25 September 2017 Accepted: 3 October 2017 
personnel. Therefore, a combination of culling and vaccination might be a better approach. Vaccination remains one of the most efficient methods to combat influenza infections. Since influenza viruses evolve rapidly over time, the closer the vaccine virus matches the circulating virus, the more protective the vaccine is. ${ }^{9}$ However, no commercial vaccines were a good match to the highly pathogenic $\mathrm{H} 5 \mathrm{~N} x$ viruses that caused the 2014-1015 outbreaks in the USA.

Newcastle disease caused by Newcastle disease virus (NDV) is an important disease of poultry for which live attenuated NDV vaccines are in wide use. Furthermore, NDV can be modified using reverse genetics and used as a vaccine platform to express one or more foreign antigens in order to develop bivalent vaccines against both NDV and other pathogens including influenza. ${ }^{10,11}$ NDV-vectored vaccines have DIVA (differentiating infected from vaccinated animals) capability for influenza and other pathogens because they express only one major antigen from target pathogen. Moreover, the attenuated NDV vaccine can be applied to large number of poultry through drinking water or aerosolization. For example, two NDV vaccines (Merck B1VAC and CLONEVAC-30) have been approved to be used by these application routes. NDV has been used as a vaccine vector for several pathogens infecting animals and humans; ${ }^{12-15}$ it has also been developed for cancer therapy. ${ }^{16}$ NDV LaSota strain is an avirulent virus and has been widely used as a vaccine in chickens. ${ }^{17}$ NDV-vectored influenza vaccines based on the LaSota strain have been demonstrated to be safe and efficacious against HPAI or LPAI virus challenge., ${ }^{9,11,18-22}$ One advantage of these recombinant vaccines is that they can be produced within four to six weeks, which is critical since influenza vaccine should be based on the circulating virus strain in the field.

In this study, a LaSota NDV-vectored $\mathrm{H} 5$ vaccine (NDV-H5) was developed based on the H5 gene of the HPAI A/chicken/lowa/0420/2015 (H5N2) virus. Subsequently, the efficacy of both live and inactivated NDV-H5 vaccines were evaluated in chickens by using the intramuscular immunization and challenging them with an HPAI A/turkey/Minnesota/9845-4/2015 (H5N2) virus. In addition, the efficacy of the live NDV-H5 vaccine was evaluated in chickens by using coarse spraying immunization and challenging them with the same HPAI H5N2 virus, and sera from vaccinated surviving birds were tested for neutralizing both HPAI H5N1 A/ American green-winged teal/ Washington /195750/2014 and H5N8 A/gyrfalcon/ Washington /41088/2014 viruses.

\section{RESULTS}

Generation and characterization of the NDV-H5 vaccine candidate The codon-optimized ectodomain of an H5 HA based on the H5N2 A/chicken/lowa/04-20/2015 virus was cloned into the NDV vector and confirmed by sequencing. The full-length cDNA clone carrying the complete antisense genome of the NDV LaSota vaccine strain with the $\mathrm{H} 5 \mathrm{HA}$ ectodomain part fused with the transmembrane and cytoplasmic tail of the NDV F protein (Fig. 1a) were cotransfected with the supporting NDV plasmids (NP, P, and L) into A549 cells. The recombinant NDV-H5 virus was rescued and amplified in SPF chicken eggs as described previously. ${ }^{23}$ Our former study demonstrated that this chimeric strategy to generate recombinant NDV $\mathrm{H} 5$ and $\mathrm{H} 7$ viruses, i.e., influenza virus $\mathrm{HA}$ ectodomain fused to the NDV $F$ protein transmembrane and cytoplasmic regions, results in efficient incorporation of the recombinant HA into the viral particle. ${ }^{18}$

To determine the HA expression by the recombinant NDV-H5 virus, we conducted immunofluorescence and flow cytometry assays using specific antibodies targeting the influenza HA and NDV antigens. Results revealed that specific green fluorescence was detected in Vero cells infected with the NDV-H5 virus using an anti-HA monoclonal antibody, but not in those infected with the wild type NDV LaSota virus (Fig. 1b); however, NDV antigens could be detected in Vero cells infected with either the NDV-H5 or the control NDV LaSota virus (Fig. 1b). Expression of HA by the recombinant NDV-H5 virus was further confirmed by flow cytometry assay (Fig. 1c). All results indicated that the recombinant NDV-H5 virus successfully expressed the H5 HA protein and could be used as a vaccine candidate for further experiments.

Both live and inactivated NDV-H5 are immunogenic in chickens To determine immunogenicity of live and inactivated NDV-H5 vaccines in chickens, both anti-H5 and anti-NDV antibody levels were tested by $\mathrm{HI}$ assay prior to vaccination, at booster vaccination (2 weeks post first vaccination) and before challenge. No antibodies against $\mathrm{H} 5$ and NDV were detected in all experimental chickens prior to vaccination. Detectable $\mathrm{HI}$ titer (1:10) against the $\mathrm{H} 5$ virus was detected only in 2 out of 20 chickens immunized with the live NDV-H5 virus at 2 weeks post the first vaccination, while 15 out of 20 birds in this group showed seroconversion $(\mathrm{HI}$ titer ranges from 10 to 80 ) after 2 weeks post booster (Fig. 2a). In contrast, 6 out of 15 chickens immunized with the inactivated NDV-H5 vaccine seroconverted at 2 weeks post the first vaccination with an $\mathrm{HI}$ titer ranging from 20 to 80 against the $\mathrm{H} 5$ virus; a significantly higher $\mathrm{HI}$ titer against the $\mathrm{H} 5$ virus was found in all birds ( $\mathrm{HI}$ titer ranges from 10 to 1280) after 2 weeks post booster (Fig. 2a). Regarding the HI titers against NDV LaSota strain virus, all vaccinated birds with either live or inactivated NDV$\mathrm{H} 5$ vaccine seroconverted at 2 weeks post the first vaccination with titers ranging from 10 to 1280 . However, the inactivated NDV-H5 induced a significantly higher titer in immunized chickens than the live NDV-H5 vaccine (Fig. 2b). Two weeks post booster, the inactivated NDV-H5 vaccinated chickens showed an increased antibody titer against NDV LaSota ranging from 160 to 1280 . In contrast, the chickens vaccinated with the live NDV-H5 virus displayed a similar or even decreased $\mathrm{HI}$ titer in most of the birds after booster vaccination; only on 6 birds which had a low titer (10-20) after the first vaccination, an increased $\mathrm{HI}$ titer was detected (Fig. 2b). These results indicated that live and inactivated NDV-H5 vaccines were immunogenic in chickens, whereas the inactivated NDV-H5 vaccine was more immunogenic in chickens than the live NDV-H5 vaccine.

Both live and inactivated NDV-H5 vaccines are able to protect chickens from lethal challenge

Chickens immunized with either the live or inactivated NDV-H5 vaccine, similar as the mock-vaccinated chickens didn't show any clinical symptoms during the vaccination period. At 2 or 3 days post challenge $(\mathrm{dpc})$ with the HPAl H5N2 virus, all mockvaccinated chickens started to show clinical signs, such as ruffled feathers, facial edema, depression, swollen and cyanotic wattles and combs, petechial hemorrhages on unfeathered skin and larger subcutaneous shank hemorrhages, and central nervous system (CNS) symptoms, and sudden death. Four birds (preassigned) were scheduled to be necropsied on $3 \mathrm{dpc}$ in order to investigate virus replication; one of them was found death on 2 $\mathrm{dpc}$, and the other 3 displayed severe symptoms when necropsied on $3 \mathrm{dpc}$. The remaining 11 birds in the mock-vaccinated group were either found dead or were humanely euthanized due to severe disease on 3 or $4 \mathrm{dpc}$. The severity of disease was scored according to clinical symptoms as shown in Fig. 3a. In contrast to mock-vaccinated birds, no immunized animals with either the live or inactivated NDV-H5 vaccine showed any clinical symptoms throughout the observation period of 14 days; all survived the lethal H5N2 challenge. These results indicated that both live and inactivated NDV-H5 vaccines were able to provide $100 \%$ protection in chickens against lethal H5N2 virus challenge (Fig. 3b). 
(b)
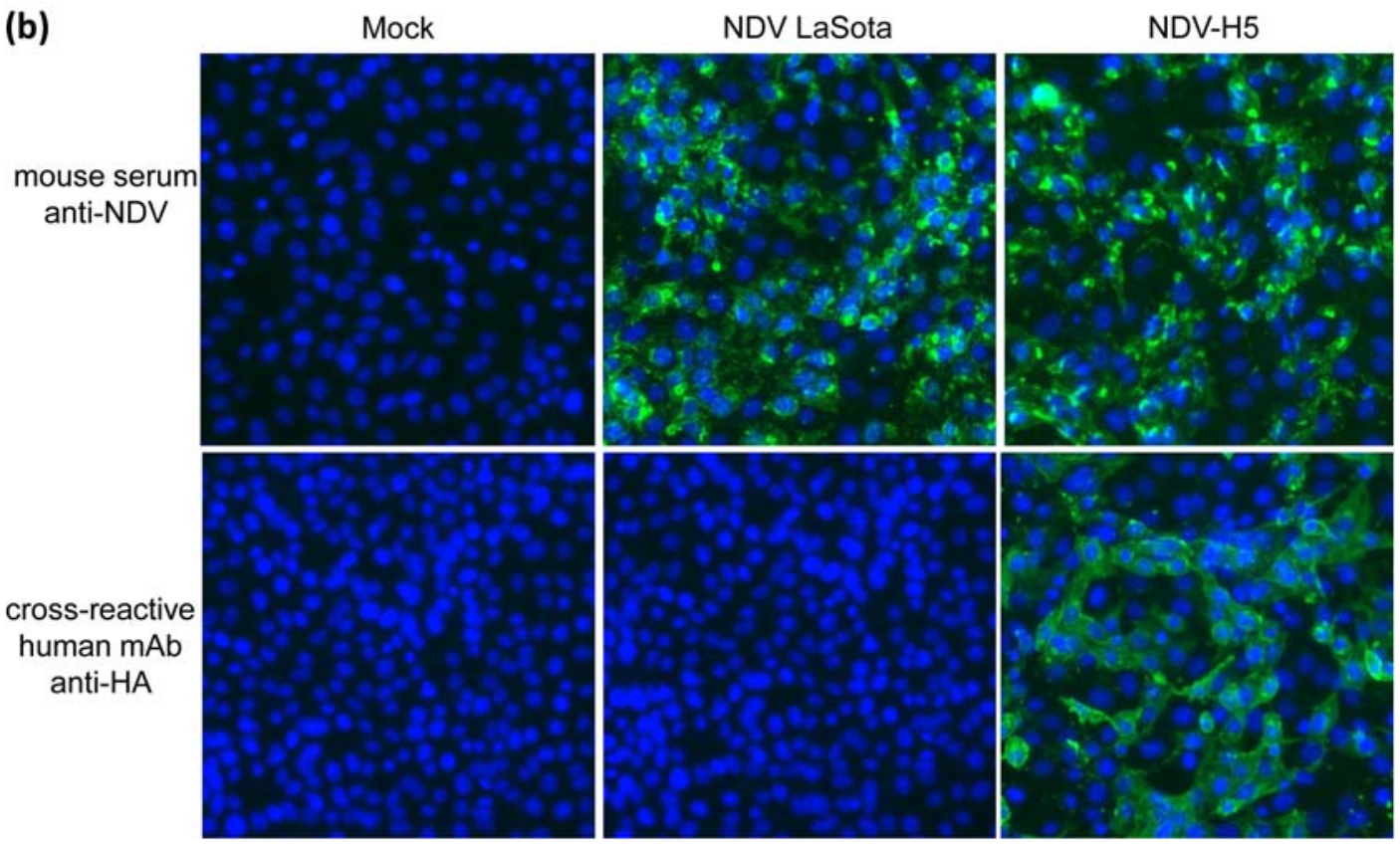

(c)

Mock cells
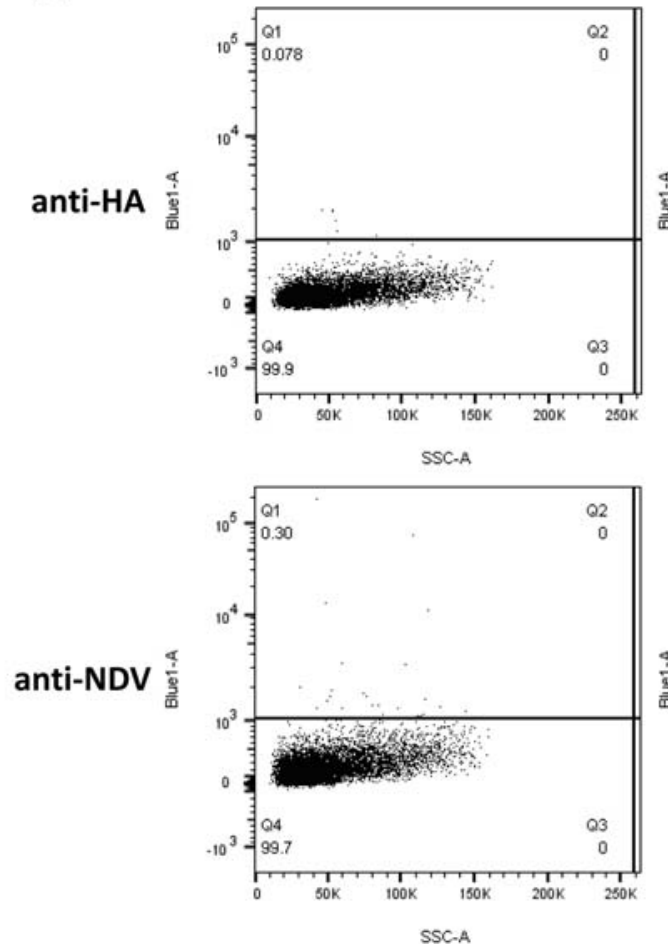

NDV-H5 infected cells
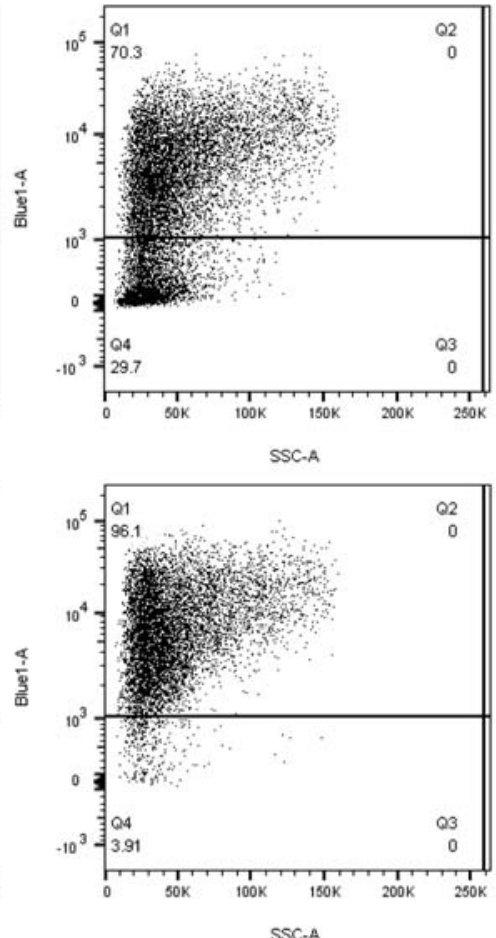

Fig. 1 Schematic design of recombinant NDV-H5 constructs and characterization of recombinant NDV-H5 virus in infected Vero cells. a The chicken codon-optimized ectodomain of hemagglutinin gene from the A/chicken/lowa/04-20/2015 (H5N2) was fused with the transmembrane and cytoplasmic tail of fusion (F) protein of NDV-LaSota strain, and cloned into the $P$ and M junction of the NDV antigenomic CDNA. The H5 ectodomain was placed under the control of a set of NDV gene start (GS) and gene end (GE) transcription signals directing its expression as a separate mRNA. b, c H5 protein was expressed and detected in NDV-H5 virus infected Vero cells using an HA human-monoclonal antibody. NDV proteins were detected in Vero cells infected with the NDV-H5 or NDV LaSota virus using mouse polyclonal NDV serum by immunofluorescence and flow cytometry assays 
(a)

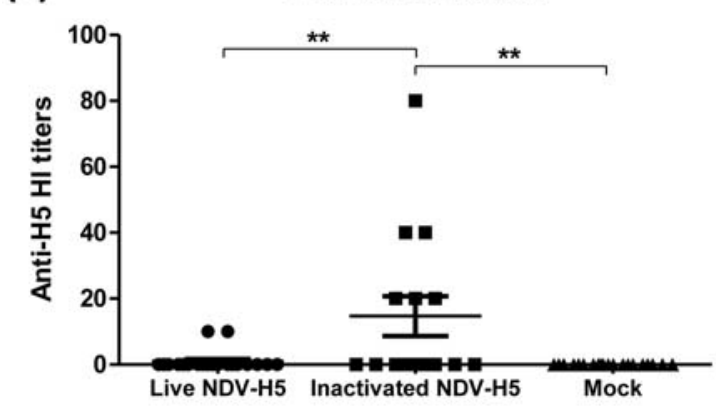

(b)

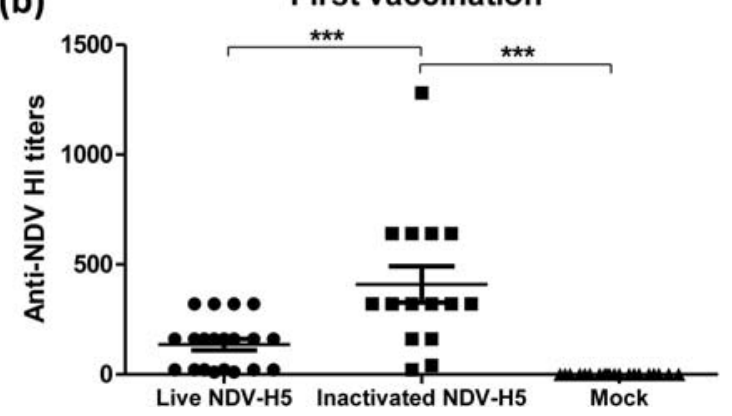

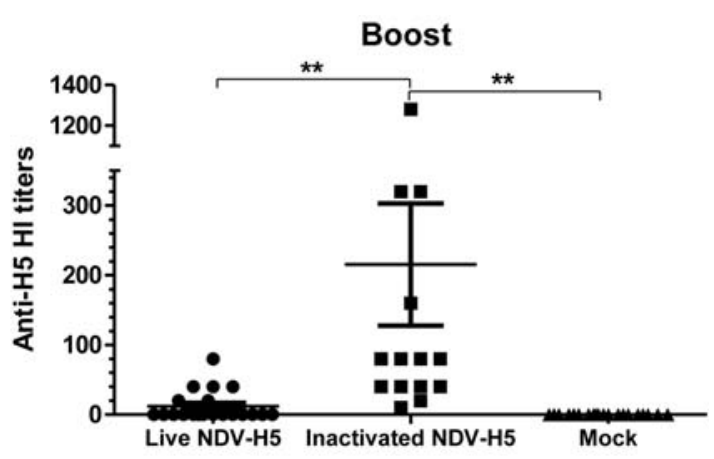

Boost

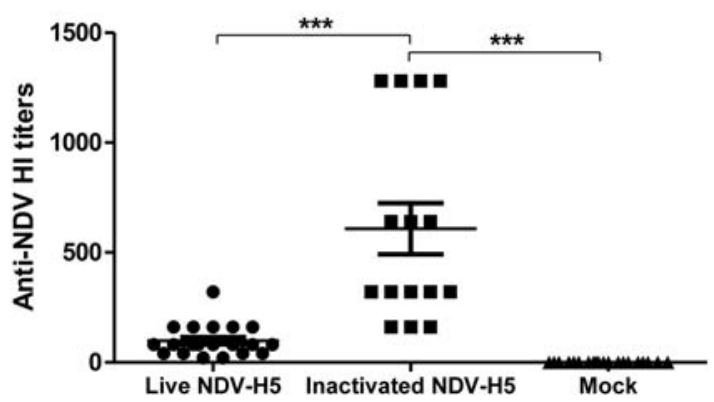

Fig. 2 Serum HI titers against $\mathrm{H} 5$ and NDV in mock-vaccinated and vaccinated chickens after two weeks of prime and booster. a Anti-H5 $\mathrm{HI}$ titer in each individual bird in each group of immunized chickens with indicated vaccine at 2 weeks post first vaccination and 2 week post boost. b Anti-NDV HI titer in each individual bird in each group of immunized chickens with indicated vaccine at 2 weeks post first vaccination and 2 week post boost. (Round dot: live NDV-H5; square: inactivated NDV-H5; triangle: mock-vaccinated)

Both live and inactivated NDV-H5 vaccines reduce viral shedding and systemic replication

To investigate whether vaccination can block virus shedding and virus replication in organs, virus titer was determined in oropharyngeal and cloacal swabs and various organs. Nine out of 15 mock-vaccinated chickens shed virus through the oropharyngeal cavity at $1 \mathrm{dpc}$, and 15 out of 15 birds at $3 \mathrm{dpc}$. No virus was detected in cloacal samples collected from mock-vaccinated chickens at $1 \mathrm{dpc}$, but virus shedding was detected in 2 out of 8 birds in cloacal swabs at $3 \mathrm{dpc}$. In contrast, virus was only found in oropharyngeal swabs collected from 1 out of 20 chickens immunized with the live NDV-H5 and 2 out of 15 birds immunized with the inactivated NDV-H5 vaccine at $1 \mathrm{dpc}$. No virus was detected in cloacal swabs and in oropharyngeal swabs collected at any other days in both live and inactivated NDV-H5 vaccinated chickens (Table 1A).

Virus replication was determined in lung, spleen and bursa fabricii collected at 3 and $5 \mathrm{dpc}$. Virus was detected in all three tissues (7/8 from lung, 5/8 from spleen, and 7/8 from bursa) collected from mock-vaccinated chickens at $3 \mathrm{dpc}$. In contrast, no virus was found in these three organs collected from chickens immunized with either the live or inactivated NDV-H5 vaccine at 3 and $5 \mathrm{dpc}$ (Table 1B). These results indicate that both live and inactivated NDV-H5 vaccines are able to reduce virus shedding and systemic replication in chickens.

Both live and inactivated NDV-H5 vaccines protect immunized chickens from pathological lesions after challenge

Histopathological (H\&E) and immunohistological (IHC) analysis of lung, spleen and bursa fabricii from vaccinated and mockvaccinated chickens challenged with the HPAI H5N2 virus revealed Influenza A virus-specific histopathology and positive IHC only in the mock-vaccinated chickens (Fig. 4). Mock-vaccinated birds had organ histopathological scores of 2 or 3 , while the vaccinated birds with either live or inactivated NDV-H5 vaccine predominantly received scores of 0 (Table 2). In the lungs of mock-vaccinated birds, the parabronchial units were more affected than the other larger airways. Tracheal and bronchial cilia were still intact but their epithelium was multifocally hyperplastic and there was mild to moderate predominantly lymphohistiocytic subepithelial inflammation. These foci had scattered influenza positive cells. The main feature of the mockvaccinated birds' lungs was a nearly diffuse heterophilic and lymphohistiocytic interstitial pneumonia accompanied by multifocal necrosis of infundibular and atrial epithelial cells and perivascular lymphocyte accumulation (Fig. 4a). In mockvaccinated chickens, there was diffuse moderate to severe lymphocytolysis within the Bursa of Fabricius follicles, both cortical and medullary, generally unaccompanied by inflammation (Fig. 4b) as well as nearly diffuse loss of splenic white pulp with marked infiltration of heterophils and pigmented macrophages (Fig. 4c). Both organs were positive for influenza NP antigen (Figs. 4b, c). In general, the chickens from both the live and inactivated vaccine groups had no histopathological lesions. Two birds from the live NDV-H5 and inactivated NDV-H5 vaccine groups, respectively, received lung scores of 1 . The one from the live NDV-H5 group had mild, multifocal subepithelial inflammation in its bronchi and the bird from the inactivated NDV-H5 group had a mild infiltration of leukocytes, predominantly histiocytes, in its parabronchial septae. Additionally, occasional cytoplasm-only positive labeling for influenza NP antigen was observed in the tracheal and large airway epithelia in several vaccinated birds, mainly in scattered histiocytes. This is in contrast to the nuclear and cytoplasmic distribution of influenza antigen found in the mock-vaccinated birds. Vaccinated birds, regardless of vaccine type, exhibited a range of histological variance in the follicles of their bursa of Fabricius that was considered to be within normal range for 7-week-old chickens. Likewise, the prominence of splenic white pulp varied for the vaccinated birds unrelated to vaccine type. 

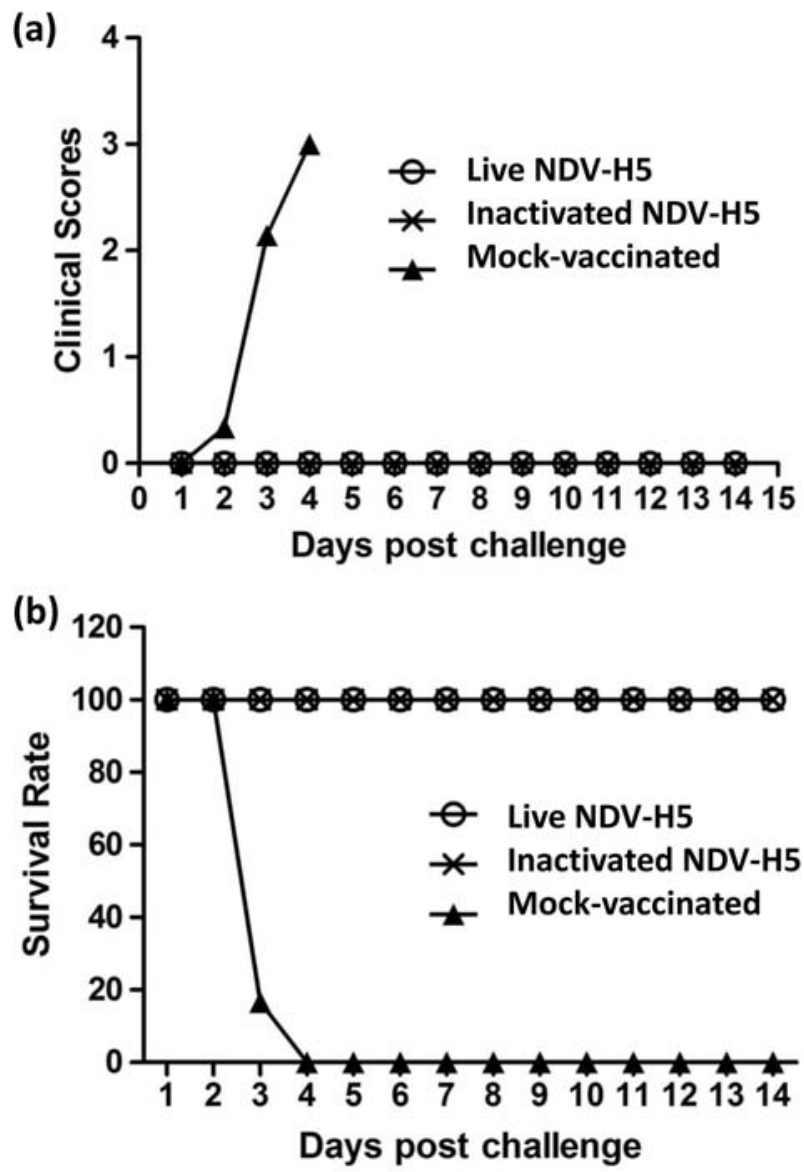

Fig. 3 Clinical scores and survival rate of vaccinated and mockvaccinated chickens after challenge. a Clinical scores were given in each group of chickens based on the clinical symptoms. The scores range from 0 to 3. (0: no clinical sign; 1: mild clinical signs; 2: severe clinical signs; 3: dead). Only the mock-vaccinated chickens showed clinical symptoms. b Survival rates of mock-vaccinated and vaccinated chickens were calculated daily. Both live and inactivated NDV-H5 vaccinated chickens survived and none of mock-vaccinated chickens survived from the challenge

One dose of the live NDV-H5 vaccine through coarse spraying immunization protects $90 \%$ chickens from the HPAI H5N2 virus challenge

None of the birds used in this study had the detectable anti-H5 titers prior to vaccination. Only 1 out of 20 chickens immunized with a single dose of live NDV-H5 by coarse spraying had the detectable anti-H5 $\mathrm{HI}$ titer $(1: 20)$ at 3 weeks post vaccination. After challenge with the HPAI H5N2 virus, mock-vaccinated chickens showed severe clinical disease. Seventeen of 20 mock-vaccinated birds were found dead at 2 or $3 \mathrm{dpc}$, and the remaining 3 birds were humanely euthanized due to severe disease at $3 \mathrm{dpc}$ (Fig. 5a, b). Only 1 out of 20 vaccinated chickens by coarse spraying was found dead at $2 \mathrm{dpc}$ and another one was found dead at $3 \mathrm{dpc}$, while the remaining birds survived until the end of the experiment (14 dpc) without clinical symptoms (Fig. 5a, b). All surviving birds seroconverted with an $\mathrm{HI}$ titer ranging from 20 to 320 (geometric mean of $\mathrm{HI}$ titer is 71) against the $\mathrm{H} 5 \mathrm{~N} 2$ virus.

At $1 \mathrm{dpc}$, no virus was detected in cloacal swabs collected from vaccinated chickens, while 6 of 20 oropharyngeal swabs were positive by virus isolation. The virus titer ranged from $10^{1.7}$ to $10^{2.5}$ $\mathrm{TCID}_{50} / \mathrm{mL}$. In contrast, virus was isolated from 5 oropharyngeal swabs (virus titers ranged from $10^{1.7}$ to $10^{2.5} \mathrm{TCID}_{50} / \mathrm{mL}$ ) and 1 cloacal swab (virus titer is $10^{3.0} \mathrm{TCID}_{50} / \mathrm{mL}$ ) collected from 20 mock-vaccinated chickens at the same date (Fig. 5c). At $3 \mathrm{dpc}$, virus was detected in oropharyngeal (virus titer is $10^{4.7} \mathrm{TCID}_{50} / \mathrm{mL}$ ) and cloacal swabs (virus titer is $10^{4.0} \mathrm{TCID}_{50} / \mathrm{mL}$ ) collected from one available mock-vaccinated birds (Fig. $5 \mathrm{c}$ ); in contrast, no virus was detected in either oropharyngeal or cloacal swabs collected from the remaining 18 vaccinated birds at 3, 5, 7, and $9 \mathrm{dpc}$ (Fig. 5c).

To investigate whether the anti-H5N2 sera from surviving birds are able to neutralize other HPAI H5 viruses, micro-neutralization (MN) assays were performed to neutralize both HPAI H5N1 A/ American green-winged teal/Washington/195750/2014 and H5N8 A/gyrfalcon/ Washington /41088/2014 viruses. All 18 surviving vaccinated chickens had MN antibody titers against both HPAl $\mathrm{H} 5 \mathrm{~N} 1$ and $\mathrm{H} 5 \mathrm{~N} 8$ viruses ranging from 80 to 1280 , with geometric mean of MN titers of 235 and 387 against the H5N1 and H5N8 viruses, respectively. Our results indicate that immunization of one dose of the live NDV-H5 vaccine by coarse spraying can provide $90 \%$ protection in vaccinated chickens, and significantly reduces virus shedding.

\section{DISCUSSION}

Eurasian-origin HPAI H5N8 virus has spread worldwide with migration of wild birds, posing a significant threat to the poultry industry. This H5N8 can readily reassort with locally endemic low pathogenic avian influenza viruses to generate novel viruses; the reassortant $\mathrm{HPAI} \mathrm{H} 5 \mathrm{~N} 1$ and $\mathrm{H} 5 \mathrm{~N} 2$ viruses detected in the USA are good examples. The reassortant $\mathrm{H} 5 \mathrm{~N} 2$ and original Eurasian H5N8 viruses have caused outbreaks in U.S. domestic birds. In order to control outbreaks of HPAI worldwide, hundreds of millions of poultry have been slaughtered to prevent the spread of the virus. The development of safe and efficacious vaccines against HPAI viruses is urgently needed to protect the poultry industry, export markets and our food supply.

In the present study, we have developed an NDV-H5 vaccine based on the NDV LaSota vaccine strain, and demonstrated that both inactivated and live NDV-H5 vaccines are able to protect chickens against a lethal HPAI H5N2 virus challenge. This was shown by protection of vaccinated chickens against clinical symptoms and mortality, by blocking virus replication in various organs and by significantly reducing viral shedding. Although both vaccines were given through the same IM route, the antibody levels of anti-HA and anti-NDV antibodies in chickens prime immunized with the inactivated NDV-H5 vaccine were much higher than in those immunized with the live NDV-H5 vaccine. This could be due to the fact that (i) the inactivated NDVH5 vaccine contained more virus antigen per dose and an adjuvant which increases the immunogenicity of vaccines ${ }^{24}$ in contrast to the live vaccine formulation, and (ii) the live NDV-H5 most likely does not replicate well in chickens through the IM immunization route as replication of the NDV LaSota strain is mainly in the mucosa. The anti-HA specific antibody levels in birds increased in both live and inactivated NDV-H5 vaccines after booster; however, the anti-NDV antibody levels decreased in the live NDV-H5 vaccine group after booster, whereas the titer in inactivated NDV-H5 vaccine group increased when compared to the titer after the first vaccination. This may indicate that preexisting NDV antibodies, such as maternal antibodies, could affect the immune responses after live NDV vaccination, ${ }^{25,26}$ whereas pre-existing NDV antibodies had almost no effect on the booster vaccination using the inactivated vaccine. Similar results have been presented in previous studies where application of a live NDV vaccine could not induce the same high levels of NDVspecific antibody levels as the inactivated vaccine when maternal antibodies were present in the birds. ${ }^{25,26}$ However, the live NDV$\mathrm{H} 5$ vaccine application was still able to provide $100 \%$ protection of chickens from the lethal $\mathrm{H} 5 \mathrm{~N} 2$ challenge. A previous study showed that a live recombinant LaSota NDV-H5 vaccine (rNDVLS/AI-H5) is efficacious in protecting chickens with high NDV- 
Table 1. Viral titers in collected swab and tissue samples from experimental chickens. (A) Viral titers in oropharyngeal and cloacal swabs collected from vaccinated and mock-vaccinated chickens at 1, 3, 5, 7, and 9 days post challenge; (B) Viral titers in different tissues collected from vaccinated or mock-vaccinated chickens at different days post challenge

\begin{tabular}{|c|c|c|c|c|c|c|c|c|c|c|c|}
\hline & \multicolumn{3}{|l|}{$1 \mathrm{dpc}$} & \multicolumn{2}{|l|}{$3 \mathrm{dpc}$} & \multicolumn{2}{|l|}{$5 \mathrm{dpc}$} & \multicolumn{2}{|l|}{$7 \mathrm{dpc}$} & \multicolumn{2}{|l|}{$9 \mathrm{dpc}$} \\
\hline & \multicolumn{2}{|c|}{ Oropharyngeal } & Cloacal & Oropharyngeal & Cloacal & Oropharyngeal & Cloacal & Oropharyngeal & I Cloacal & Oropharyngeal & Cloacal \\
\hline \multicolumn{12}{|l|}{ (A) } \\
\hline $\begin{array}{l}\text { Inactivated } \\
\text { NDV-H5 }\end{array}$ & \multicolumn{2}{|c|}{$\begin{array}{l}1.7^{\mathrm{a}}(1 / 20)^{\mathrm{b}} \\
2.5 \pm 0^{\mathrm{a}}(2 / 15)^{\mathrm{b}}\end{array}$} & $<1.0$ & $<1.0$ & $<1.0$ & $<1.0$ & $<1.0$ & $<1.0$ & $<1.0$ & $<1.0$ & $<1.0$ \\
\hline \multirow{3}{*}{$\begin{array}{l}\text { Mock- } \\
\text { vaccinated }\end{array}$} & \multicolumn{2}{|c|}{$2.43 \pm 0.18^{\mathrm{a}}(9 / 15)^{\mathrm{b}}$} & $<1.0$ & $3.18 \pm 0.31^{a}(8 / 8)^{b}$ & $1.7 \pm 0^{\mathrm{a}}(2 / 8)^{\mathrm{b}}$ & $\mathrm{N} / \mathrm{A}$ & $N / A$ & $\mathrm{~N} / \mathrm{A}$ & $\mathrm{N} / \mathrm{A}$ & $\mathrm{N} / \mathrm{A}$ & $\mathrm{N} / \mathrm{A}$ \\
\hline & & \multicolumn{4}{|c|}{ Lung } & \multicolumn{3}{|l|}{ Spleen } & \multicolumn{3}{|l|}{ Bursa } \\
\hline & & \multicolumn{2}{|c|}{$3 \mathrm{dpc}$} & & $5 \mathrm{dpc}$ & $3 \mathrm{dpc}$ & & $5 \mathrm{dpc}$ & \multicolumn{2}{|l|}{$3 \mathrm{dpc}$} & $5 \mathrm{dpc}$ \\
\hline \multicolumn{12}{|l|}{ (B) } \\
\hline \multicolumn{2}{|l|}{ Live NDV-H5 } & \multicolumn{2}{|l|}{$<1.0$} & \multicolumn{2}{|l|}{$<1.0$} & \multicolumn{2}{|l|}{$<1.0$} & $<1.0$ & \multicolumn{2}{|l|}{$<1.0$} & $<1.0$ \\
\hline \multicolumn{2}{|c|}{ Inactivated NDV-H5 } & \multicolumn{2}{|l|}{$<1.0$} & \multicolumn{2}{|l|}{$<1.0$} & \multicolumn{2}{|l|}{$<1.0$} & $<1.0$ & \multicolumn{2}{|l|}{$<1.0$} & $<1.0$ \\
\hline
\end{tabular}

specific maternal antibodies against challenge with a virulent HPAI $\mathrm{H} 5$ virus and a velogenic NDV strain. ${ }^{27}$ These results suggest that the live NDV-H5 vaccine could also overcome the interference of existing NDV-specific maternal antibodies. This question needs to be investigated in future studies.

Furthermore, we have performed a study using a Pump-up Sprayer to massively apply the live NDV-H5 vaccine via coarse spraying in order to test the protective efficacy using a labor costeffective immunization strategy. Results showed that the engineered live NDV-H5 vaccine is able to provide $90 \%$ protection to chickens through one single dose of spray vaccination. It should be noted that failure to provide complete protection in immunized birds is probably due to following two reasons: (1) immunization is taken up unevenly as two chickens displayed severe symptoms at 2-3 dpc, indicating that these birds probably were not exposed to the vaccine; (2) a Pump-up Sprayer used for vaccination produced droplets of size approximately $300 \mu$, which will affect vaccine inhalation through nasal cavity and mucous absorption through the eyes. We suggest that protection efficacy of the NDV-H5 vaccine could be improved if an ideal instrument for coarse spray immunization are designed to produce correct size of droplets. Future research avenues will investigate if this is the case. In any case, our studies here indicate that the newly developed NDV-H5 vaccine could be used to combat outbreaks caused by the intercontinental $\mathrm{H} 5 \mathrm{~N} x$ viruses.

It should be noted that the vaccination regimen used, i.e., timing between vaccinations ( 2 weeks between prime and boost for the intramuscular immunizations) and challenge is relatively short (2-3 weeks) in both our studies. This indicates that the vaccine is effective even after a few weeks post-vaccination. However, this timing does not allow the evaluation of long term protection. In order to evaluate how long the immune responses induced by the developed NDV-H5 vaccine will last, challenges should be conducted later such as 8 or more weeks post vaccination. Therefore, additional studies are needed to determine long-term protection of the developed NDV-H5 vaccine. HPAI H5 viruses cause severe impacts not only on chickens, but also on turkeys and domesticated waterfowls. We have demonstrated that the developed NDV-H5 vaccine is able to provide efficient protection in immunized chickens. The efficacy of the NDV-H5 vaccine in other species, such as turkeys, ducks and geese, will be evaluated in our future studies.

It is important to note that we are able to produce the recombinant NDV-vectored influenza vaccine with DIVA capability within 4-6 weeks after we receive the HA sequence of a particular influenza strain. This is important since influenza vaccines have to be updated regularly based on the circulating or newly emerging virus strain. Therefore, an NDV vector-based influenza vaccine could be produced as fast as a conventional inactivated influenza virus vaccine, with the advantage that the NDV-based vaccine can be applied by coarse spraying. A previous study has shown that an NDV-vectored influenza $\mathrm{H} 5$ vaccine is able to induce high titer of serum neutralizing antibodies against HPAI H5 virus in a nonhuman primate model, the African green monkey. ${ }^{28}$ This indicates that NDV-vectored influenza vaccines most likely could also be used to vaccinate mammalian species against influenza viruses. In this aspect, it should be noted that a NDV-vectored rabies vaccine has been shown to be safe, highly immunogenic, to provide longlasting protection in dogs and cats. ${ }^{29}$ All these data indicate that the NDV-vectored influenza vaccines might be efficacious in various mammalian hosts including humans against influenza infection. Since NDV is not a mammalian pathogen, this approach should be safe in mammals.

\section{METHODS}

Ethics statements

All animal studies were approved and carried out in strict accordance to the recommendations in the guidelines of the Institutional Animal Care and Use Committee at Kansas State University, an AAALAC institution. All researches related to highly pathogenic $\mathrm{H} 5$ viruses were performed in biosafety level 3 laboratory and facilities (BSL3) in the Biosecurity Research Institute at Kansas State University.

Viruses, cell culture, and eggs

HPAI H5 viruses including A/chicken/lowa/04-20/2015 (H5N2), A/turkey/ Minnesota/9845-4/2015 (H5N2), A/American green-winged teal/Washington/195750/2014 (H5N1) and A/gyrfalcon/Washington/41088/2014 (H5N8) were kindly provided by the National Veterinary Services Laboratories (NVSL) at Ames, IA and propagated in ten-day-old specific-pathogen-free (SPF) embryonated chicken eggs. Mardin-Darby Canine Kidney Cells 
(a)

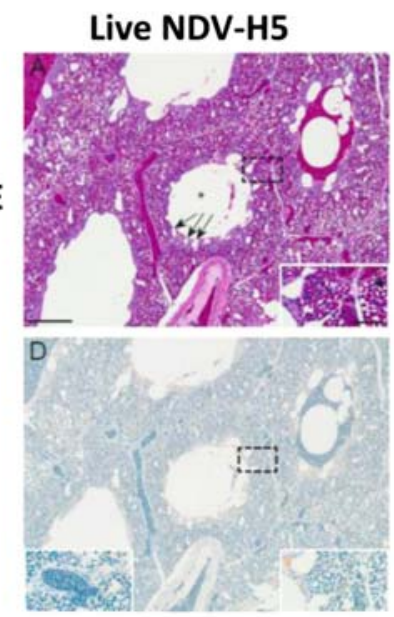

(b)

H\&E

IHC
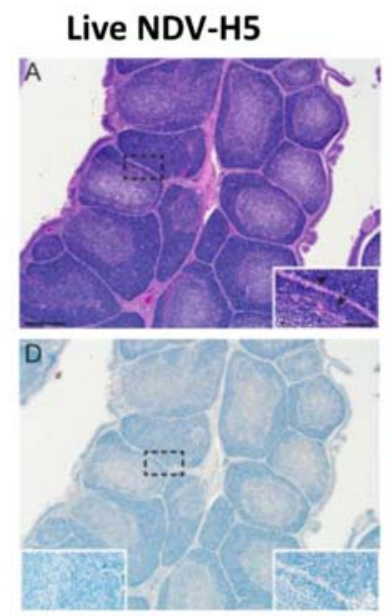

(c)
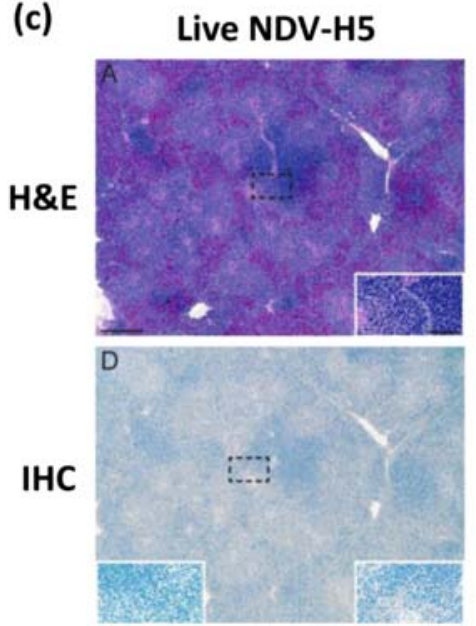

inactivated NDV-H5
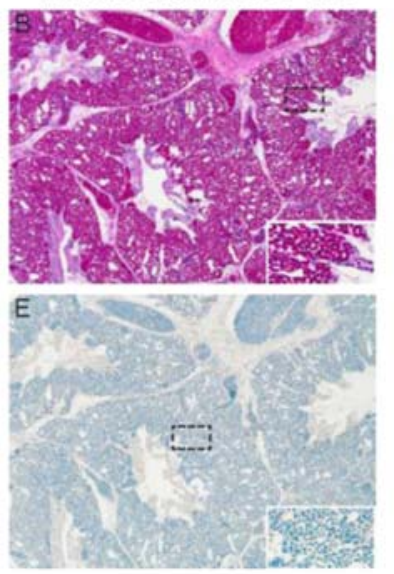

inactivated NDV-H5
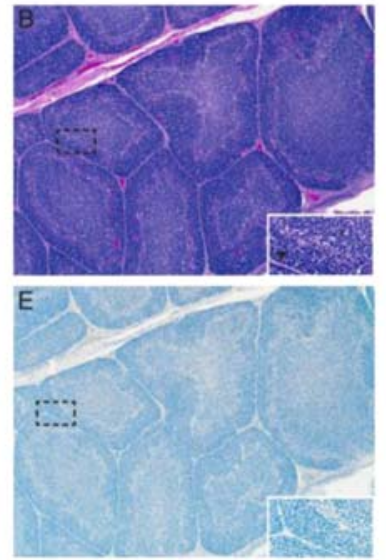

inactivated NDV-H5
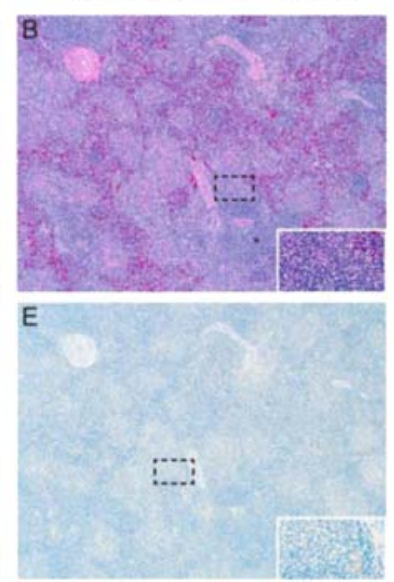

Mock-vaccinated

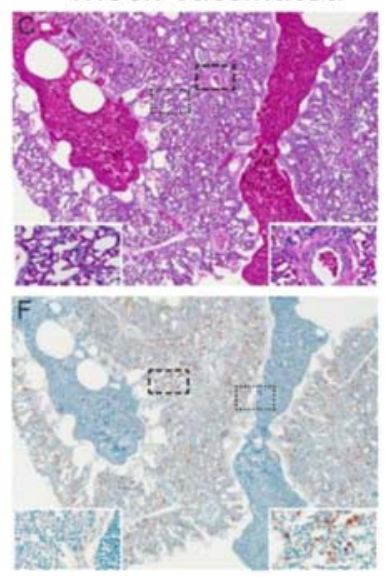

\section{Mock-vaccinated}
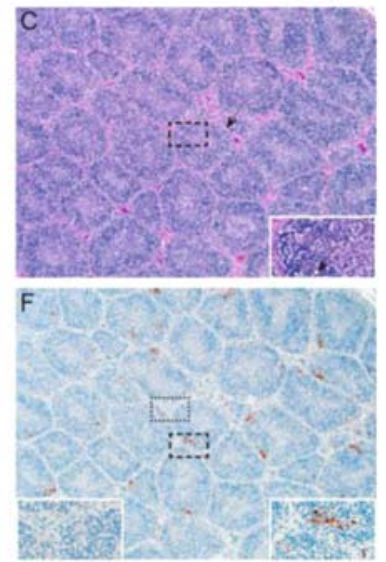

Mock-vaccinated
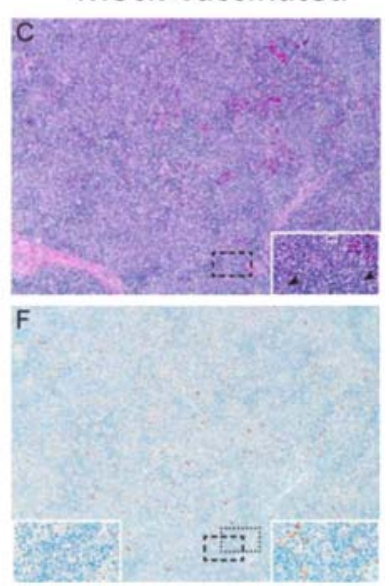

Fig. 4 Histopathology and immunochemistry panel of lung, spleen, and bursa of vaccinated and mock-vaccinated chickens at 3 dpc. a H\&E and IHC staining in the lungs of chickens that were vaccinated or mock-vaccinated as indicated. $\mathbf{b}$ H\&E and IHC staining in the bursa of birds that were vaccinated or mock-vaccinated as indicated. c H\&E and IHC staining in the spleens of birds that were vaccinated or mock-vaccinated as indicated

(MDCK) were maintained in Minimum Essential Medium (MEM) supplemented with $5 \%$ fetal bovine serum (FBS) (HyClone; Logan UT), 1x Lglutamine (Invitrogen, Carlsbad, CA), 1x MEM vitamins (Invitrogen; Carlsbad, CA), and $1 \mathrm{x}$ antibiotics (Invitrogen, Carlsbad, CA) under $37^{\circ} \mathrm{C}$ with $5 \% \mathrm{CO}_{2}$. Adenocarcinomic human alveolar basal epithelial cells (A549) were used for transfection of NDV anti-genome cDNA with NDV supporting plasmids expressing NDV N, $P$, and $L$ to generate recombinant NDV viruses. African green monkey kidney (Vero) and A549 cells were cultured in Dulbecco's Modified Eagle Medium (DMEM) maintained and supplemented with 10\% FBS and other reagents as the MDCK cells 
Table 2. Histopathology scores of tissues collected from vaccinated and mock-vaccinated chickens at 3 and $4 / 5 \mathrm{dpc}$

\begin{tabular}{|c|c|c|c|c|c|c|}
\hline & \multicolumn{3}{|l|}{$3 \mathrm{dpc}$} & \multicolumn{3}{|c|}{$4 / 5^{\mathrm{a}} \mathrm{dpc}$} \\
\hline Live NDV-H5 & $0(0 / 5)$ & $0(0 / 5)$ & $0.2 \pm 0.22(1 / 5)^{b}$ & $0(0 / 5)$ & $0(0 / 5)$ & $0(0 / 5)$ \\
\hline Inactivated NDV-H5 & $0(0 / 4)$ & $0(0 / 4)$ & $0.25 \pm 0.25(1 / 4)^{b}$ & $0(0 / 4)$ & $0(0 / 4)$ & $0(0 / 4)$ \\
\hline Mock-vaccinated $^{c}$ & $2.8 \pm 0.2(5 / 5)^{d}$ & $2.6 \pm 0.24(5 / 5)^{d}$ & $2.8 \pm 0.2(5 / 5)^{d}$ & $3(1 / 1)^{c}$ & $3(1 / 1)^{c}$ & $2(1 / 1)^{c}$ \\
\hline
\end{tabular}

(a)

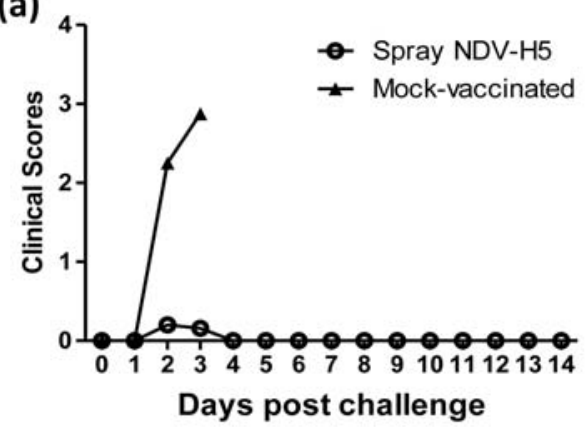

(c)

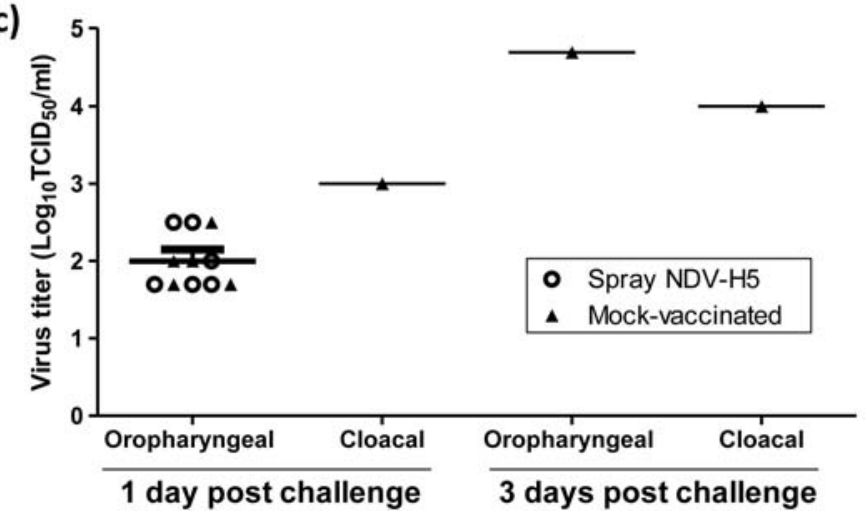

(b)

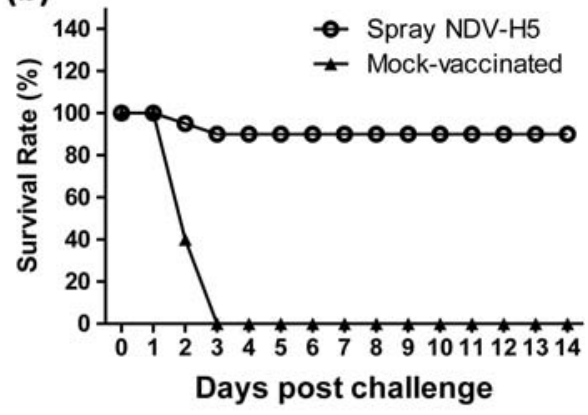

3 days post challenge

Fig. 5 Clinical scores, survival rate and virus shedding of spray vaccinated and mock-vaccinated chickens after challenge. a Clinical scores were given in each group of chickens based on the clinical symptoms. The scores range from 0 to 3 . (0: no clinical sign; 1 : mild clinical signs; 2 : severe clinical signs; 3: dead). All mock-vaccinated chickens and only 2 vaccinated birds showed clinical symptoms. b Survival rates of mockvaccinated and vaccinated chickens were calculated daily. Eighteen NDV-H5 vaccinated chickens survived and none of mock-vaccinated chickens survived from the challenge. c Viral titers in oropharyngeal and cloacal swabs collected from vaccinated and mock-vaccinated chickens at 1 and 3 days post challenge

described above. Virus titers were determined on 96-well MDCK cells and $\mathrm{TCID}_{50}$ per milliliter was calculated by the Reed and Muench method. ${ }^{30,31}$

\section{Vaccine construction and preparation}

Newcastle disease virus-vectored (NDV-vectored) vaccine was constructed by expressing the codon optimized ectodomain of the $\mathrm{H} 5$ hemagglutinin (HA) gene from the A/chicken/lowa/04-20/2015 (H5N2) virus (Genbank accession number KR492974.3). The multiple basic cleavage site (RERRRKR/ GLF) of $\mathrm{H} 5$ was replaced with a monobasic cleavage site (ESR/GLF). The optimized sequence coding the ectodomain of the HA was fused in frame to the sequence coding the trans-membrane and cytoplasmic tail of the NDV fusion protein and subsequently cloned between the $P$ and $M$ genes of a full length CDNA of the NDV vaccine strain LaSota as described previously. ${ }^{18}$ Flanking NDV's gene end (GE), intergenic (IS), and gene start (GS) regulatory sequences were inserted to ensure recognition as an additional viral gene. A Kozak sequence was inserted upstream of the start codon for optimal initiation of translation (Fig. 1a). The recombinant NDV virus expressing the H5 HA (NDV-H5) was rescued by reverse genetics, then amplified in SPF embryonated chicken eggs for further experiments.

To verify if the H5 HA protein was expressed by the recombinant NDV$\mathrm{H} 5$ virus, Vero cells were infected with the NDV-H5 virus and fixed at 24 $\mathrm{hpi}$, and immunofluorescence assay was performed to detect HA protein using a human monoclonal antibody CR9114 ${ }^{32}$ that recognizes a conserved epitope in the stalk domain of the HA from influenza A and B viruses. To detect NDV antigens, we used a mouse-origin anti-NDV polyclonal serum prepared by pooling the serum of 5 mice infected with the wild type NDV, LaSota strain. Fluorescently labeled anti-human IgG and anti-mouse IgG (Invitrogen) were used as secondary antibodies. Flow cytometry was used to confirm HA protein expression by NDV-H5 virus infected Vero cells using the same anti-HA monoclonal antibody. The wildtype NDV LaSota strain was used as a control for both assays. 
The amplified recombinant NDV-H5 virus was concentrated by ultracentrifugation using a $30 \%$ sucrose cushion. The concentrated virus was titrated and inactivated by the UV for $1 \mathrm{~h}$. The inactivated virus was mixed with $15 \%$ water-in-oil adjuvant (ISA 70, Seppic) to make inactivated $\mathrm{NDV}-\mathrm{H} 5$ vaccine. Inactivated NDV-H5 vaccine contains the equivalent to $10^{7} \mathrm{TCID}_{50}$ of NDV-H5 virus per dose prior to inactivation.

\section{Chicken experiments}

To evaluate the efficacy of the developed NDV-H5 vaccine, two experiments using two-week-old SPF White Leghorn chickens (purchased from Charles River Laboratories) were performed. In the first experiment, the NDV-H5 vaccine was administered through the intramuscular (i.m.) route, not through the oculonasal route, because our pervious study showed that the antibody levels of chickens vaccinated intramuscularly were higher than those of chickens vaccinated oculonasally. ${ }^{18}$ Fifty chickens were randomly separated to three groups. Twenty chickens in the group 1 were intramuscularly immunized with $5 \times 10^{6} \mathrm{TCID}_{50}$ of live $\mathrm{NDV}-\mathrm{H} 5$ vaccine per bird in $200 \mu \mathrm{l}$. Fifteen chickens in the group 2 were immunized with the inactivated NDV-H5 vaccine $\left(10^{7} \mathrm{TCID}_{50}\right.$ in $200 \mu \mathrm{l}$ per chicken) through the i.m. route. The remaining 15 chickens were intramuscularly inoculated with $200 \mu \mathrm{l}$ of phosphate-buffered saline (PBS) as mock-vaccinated controls. Two weeks after the first vaccination, chickens were boosted with the same vaccine using the same procedure as that of the first vaccination. Two weeks after the booster, all chickens were oculonasally challenged with $10^{6} \mathrm{TCID}_{50}$ of an HPAI A/turkey/ Minnesota/9845-4/2015 (H5N2) virus in $200 \mu$ l. Chickens were bled to collect serum samples prior to each vaccination and challenge. Oropharyngeal and cloacal swabs were collected on $0,1,3,5,7,9,11$, and 13 days post challenge $(\mathrm{dpc})$. Clinical signs were monitored daily. Five chickens in the live NDV-H5 immunization group, 4 chickens in the NDV-H5 inactivated vaccine immunization group, and 4 chickens in mock-vaccinated control group were predesignated to be euthanized on 3 and $5 \mathrm{dpc}$. When a necropsy designated bird was found dead prematurely or if a bird met study end point criteria for euthanasia, they were necropsied at that time point instead. At necropsy the lungs, spleen, and the bursa of Fabricius were collected from each bird. A piece from each organ was frozen for virological analysis and the remainder was placed in $10 \%$ neutral buffered formalin for histopathological analysis. The remaining birds in each group were monitored for 14 days in order to determine vaccine efficacy. At 14 $\mathrm{dpc}$, all surviving birds were euthanized. Sera were collected from all necropsied chickens, and hemagglutination inhibition ( $\mathrm{HI}$ ) assay was performed to determine seroconversion.

In the second experiment, 40 two-week-old chickens were divided into two groups. Twenty chickens were immunized with the live NDV-H5 vaccine by using a coarse sprayer (Pump-up Sprayer \#28584, Valley Vet Supply, Marysville, Kansas) to spray chicken feathers and bodies when they grouped together in a plastic box. Each bird was predicted to get $10^{6}$ $\mathrm{TCID}_{50}$ of the live NDV-H5 virus through contacting the aerosol produced by the coarse spraying. The remaining 20 chickens were sprayed with PBS and used as mock-vaccinated controls. Each chicken was challenged with $10^{6} \mathrm{TCID}_{50}$ of the HPAl A/turkey/Minnesota/9845-4/2015 (H5N2) as described above at 21 days post vaccination. Chickens were bled to collect serum samples before vaccination and challenge. In addition, the challenged chickens were observed daily to monitor clinical signs and mortality. Oropharyngeal and cloacal swabs were collected at 0, 1, 3, 5, 7, and $9 \mathrm{dpc}$ to determine viral shedding. Serum samples were collected from all surviving chickens prior to euthanasia at $14 \mathrm{dpc}$ to perform the microneutralization assay to determine cross-reactions with both HPAI H5N1 A/ American green-winged teal/WA/195750/2014 and H5N8 A/gyrfalcon/WA/ $41088 / 2014$ viruses based on the standard protocol.

\section{Histopathology and immunohistochemistry}

Standard hematoxylin and eosin (H\&E) slide staining for the collected tissues was performed and influenza nucleoprotein (NP) was detected in the tissues by immunohistochemistry $(\mathrm{IHC})$ assay using a Rabbit Anti-H1N1 NP polyclonal antibody (GenScript, NJ) as described in our prior study. ${ }^{18}$ H\&E stained tissues and immunohistochemistry were reviewed by a veterinary pathologist in a blinded fashion. Histopathology for all necropsied birds was semi-quantitatively scored for each organ on a scale of $0-3$ unless prevented by autolysis. For all tissues a score of 0 was within normal limits. For the lungs: score 1 was increased interstitial lymphocytes and histiocytes, score 2 was mild to moderate multifocal heterophilic and lymphistiocytic interstitial pneumonia; multifocal, mild to moderate lymphohistiocytic bronchitis and tracheitis; multifocally, low numbers of perivascular lymphocytes and score 3 was moderate to severe, diffuse heterophilic and lymphohistiocytic interstitial pneumonia; multifocal to nearly diffuse moderate tracheitis and bronchitis. For the spleen: score 1 was occasional foci of splenic necrosis in the white pulp, score 2 was multifocal lymphoid depletion, splenic necrosis, and mild histiocytic and heterophilic splenitis, and score 3 was nearly diffuse lymphoid depletion, splenic necrosis, and moderate to severe histiocytic and heterophilic splenitis with marked hyperplasia of phagocytic macrophage cells. For the bursa of Fabricius: score 1 was cortical or medullary lymphoid depletion and/or mild interstitial or epithelial inflammation, score 2 was moderate follicular lymphocytolysis, cortical and medullary optionally accompanied by inflammation, multifocal loss of distinction between medulla and cortex, and score 3 was severe follicular lymphocytolysis, cortical and medullary, loss of cortical medullary distinction, pooling of proteinaceous material to formation of cysts in the medulla, optionally accompanied by inflammation. IHC results were recorded as presence or absence of influenza antigen in each organ in predesignated $3 \mathrm{dpc}$ birds only. All microscopic images were captured with a BX46 light microscope equipped with a DP25 camera (Olympus; Tokyo, Japan) using CellSens Standard version 1.12 (Olympus) then further color calibrated using ChromaCal software version 2.5 (Datacolor Inc., Lawrenceville, $\mathrm{NJ}$ ) as per manufacturer's instructions.

\section{Statistical analysis}

Virus titers and antibody titers among groups were analyzed using Oneway ANOVA in GraphPad Prism version 6.0 (Graph-Pad Software Inc., CA). Those response variables were subjected to comparisons for all pairs by using the Tukey-Kramer test. Pairwise mean comparisons between vaccinated and mock groups were made using the Student $t$-test. A $p$ value of $\leq 0.05$ was considered as a significant difference.

\section{Data availability statements}

The datasets generated during and/or analyzed during the current study are available from the corresponding authors on reasonable request.

\section{ACKNOWLEDGEMENTS}

We thank Tammy Koopman, Yonghai Li, Chester McDowell, Abdou Nagy, Dingping Bai, Vinay Shivanna, Aaron Balogh, Abaineh Endalew, David Meekins, Kinga Urbaniak, Dashzeveg Bold, Rachel Palinski and Jessie Trujillo for helping with the chicken experiments, and also thank Dr. Mia Kim from APHIS National Veterinary Services Laboratories for providing both A/chicken/lowa/04-20/2015 (H5N2), A/turkey/ Minnesota/9845-4/2015 (H5N2), A/American green-winged teal/Washington/ $195750 / 2014$ (H5N1) and A/gyrfalcon/Washington/41088/2014 (H5N8) viruses and Dr. Florian Kramer from the Icahn School of Medicine at Mount Sinai for providing the anti-HA monoclonal antibody CR9114. This work was supported by KBA/CEEZAD funds, by NIAID funded Centers of Excellence for Influenza Research and Surveillance, under contract numbers HHSN266200700006C and HHSN272201400008C, and by NBAF transition and Avimex funds.

\section{AUTHOR CONTRIBUTIONS}

W.M., J.A.R, and A.G-S. conceived and designed the experiments; J.M., J.L., H.L., I.M., S. D., S.Y.S., Y.L., M.D., I.M., Y.L., J.Y., and W.M. performed the experiments and analyzed the data; J.M., J.L., A.G-S., J.A.R., and W.M. wrote and approved the completed manuscript.

\section{ADDITIONAL INFORMATION}

Competing interests: A.G-S is an inventor of patents encompassing NDV-vectored vaccines owned by the Icahn School of Medicine at Mount Sinai. The remaining authors declare that they have no Competing financial interests.

Publisher's note: Springer Nature remains neutral with regard to jurisdictional claims in published maps and institutional affiliations.

\section{REFERENCES}

1. Lee, Y. J. et al. Novel reassortant influenza A(H5N8) viruses, South Korea, 2014. Emerg. Infect. Dis. 20, 1087-1089 (2014).

2. Smith, G. J. \& Donis, R. O. Nomenclature updates resulting from the evolution of avian influenza $\mathrm{A}(\mathrm{H} 5)$ virus clades 2.1.3.2a, 2.2.1, and 2.3.4 during 2013-2014. Influenza Other Respir. Viruses 9, 271-276 (2015). 
3. Pasick, J. et al. Reassortant highly pathogenic influenza A H5N2 virus containing gene segments related to Eurasian H5N8 in British Columbia, Canada, 2014. Sci. Rep. 5, 9484 (2015).

4. Jhung, M. A. \& Nelson, D. I. Outbreaks of avian influenza A (H5N2), (H5N8), and (H5N1) among birds-United States, December 2014-January 2015. MMWR 64, 111 (2015).

5. Highly pathogenic avian influenza spreads in the USA. Vet. Rec. 176, 505 (2015).

6. USDA. Update on Avian Influenza Findings Poultry Findings Confirmed by USDA's National Veterinary Services Laboratories. (2015).

7. Ip, H. S. et al. Novel Eurasian highly pathogenic avian influenza A H5 viruses in wild birds, Washington, USA, 2014. Emerg. Infect. Dis. 21, 886-890 (2015).

8. Torchetti, M. K. et al. Novel H5 Clade 2.3.4.4 reassortant $(\mathrm{H} 5 \mathrm{~N} 1)$ virus from a green-winged teal in Washington, USA. Genome Announc. 3, e00195-15 (2015).

9. Nayak, B. et al. Immunization of chickens with Newcastle disease virus expressing $\mathrm{H} 5$ hemagglutinin protects against highly pathogenic $\mathrm{H} 5 \mathrm{~N} 1$ avian influenza viruses. PLOS ONE 4, e6509 (2009).

10. Khattar, S. K. et al. Mucosal immunization with Newcastle disease virus vector coexpressing HIV-1 Env and Gag proteins elicits potent serum, mucosal, and cellular immune responses that protect against vaccinia virus Env and Gag challenges. mBio. 6, e01005 (2015).

11. Kim, S. H., Paldurai, A., Xiao, S., Collins, P. L. \& Samal, S. K. Modified Newcastle disease virus vectors expressing the $\mathrm{H} 5$ hemagglutinin induce enhanced protection against highly pathogenic $\mathrm{H} 5 \mathrm{~N} 1$ avian influenza virus in chickens. Vaccine 32, 4428-4435 (2014).

12. Wang, J. et al. Generation and evaluation of a recombinant genotype VII Newcastle disease virus expressing VP3 protein of Goose parvovirus as a bivalent vaccine in goslings. Virus Res. 203, 77-83 (2015).

13. Zhao, W. et al. Newcastle disease virus (NDV) recombinants expressing infectious laryngotracheitis virus (ILTV) glycoproteins $\mathrm{gB}$ and gD protect chickens against ILTV and NDV challenges. J. Virol. 88, 8397-8406 (2014).

14. Kanabagatte, B. M. et al. A recombinant Newcastle disease virus (NDV) expressing infectious laryngotracheitis virus (ILTV) surface glycoprotein D protects against highly virulent ILTV and NDV challenges in chickens. Vaccine 32, 3555-3563 (2014).

15. Huang, Z., Elankumaran, S., Yunus, A. S. \& Samal, S. K. A recombinant Newcastle disease virus (NDV) expressing VP2 protein of infectious bursal disease virus (IBDV) protects against NDV and IBDV. J. Virol. 78, 10054-10063 (2004).

16. Vigil, A., Martinez, O., Chua, M. A. \& Garcia-Sastre, A. Recombinant Newcastle disease virus as a vaccine vector for cancer therapy. Mol. Ther. 16, 1883-1890 (2008).

17. Nakaya, T. et al. Recombinant Newcastle disease virus as a vaccine vector. J. Virol. 75, 11868-11873 (2001).

18. Liu, Q. et al. Newcastle disease virus-vectored $\mathrm{H} 7$ and $\mathrm{H} 5$ live vaccines protect chickens from challenge with $\mathrm{H} 7 \mathrm{~N} 9$ or $\mathrm{H} 5 \mathrm{~N} 1$ avian influenza viruses. J. Virol. 89, 7401-7408 (2015)

19. Schroer, D. et al. Efficacy of Newcastle disease virus recombinant expressing avian influenza virus $\mathrm{H} 6$ hemagglutinin against Newcastle disease and low pathogenic avian influenza in chickens and turkeys. Avian Dis. 55, 201-211 (2011).

20. Nagy, A. et al. Recombinant Newcastle disease virus expressing H9 HA protects chickens against heterologous avian influenza H9N2 virus challenge. Vaccine 34, 2537-2545 (2016).
21. Ferreira, H. L. et al. Comparison of single 1-day-old chick vaccination using a Newcastle disease virus vector with a prime/boost vaccination scheme against a highly pathogenic avian influenza H5N1 challenge. Avian Pathol. 43, 68-77 (2014).

22. Lardinois, A. et al. Potency of a recombinant NDV-H5 vaccine against various HPAI H5N1 virus challenges in SPF chickens. Avian Dis. 56, 928-936 (2012).

23. Ayllon, J., Garcia-Sastre, A. \& Martinez-Sobrido, L. Rescue of recombinant Newcastle disease virus from cDNA. J. Vis. Exp. 11, e50830 (2013).

24. Volkova, M. A. et al. Adjuvant effects of chitosan and calcium phosphate particles in an inactivated Newcastle disease vaccine. Avian Dis. 58, 46-52 (2014).

25. Facon, C., Guerin, J. L. \& Lacroix, F. Assessment of newcastle disease vaccination of houbara bustard breeders (Chlamydotis undulata undulata). J. Wildl. Dis. 41, 768-774 (2005).

26. Ferreira, H. L. et al. Immune responses and protection against $\mathrm{H} 5 \mathrm{~N} 1$ highly pathogenic avian influenza virus induced by the Newcastle disease virus H5 vaccine in ducks. Avian Dis. 56, 940-948 (2012).

27. Sarfati-Mizrahi, D. et al. Protective dose of a recombinant Newcastle disease LaSota-avian influenza virus $\mathrm{H} 5$ vaccine against $\mathrm{H} 5 \mathrm{~N} 2$ highly pathogenic avian influenza virus and velogenic viscerotropic Newcastle disease virus in broilers with high maternal antibody levels. Avian Dis. 54, 239-241 (2010).

28. DiNapoli, J. M. et al. Immunization of primates with a Newcastle disease virusvectored vaccine via the respiratory tract induces a high titer of serum neutralizing antibodies against highly pathogenic avian influenza virus. J. Virol. 81, 11560-11568 (2007).

29. Ge, J. et al. Newcastle disease virus-vectored rabies vaccine is safe, highly immunogenic, and provides long-lasting protection in dogs and cats. J. Virol. 85, 8241-8252 (2011).

30. Klimov, A. et al. Influenza virus titration, antigenic characterization, and serological methods for antibody detection. Methods Mol. Biol. 865, 25-51 (2012).

31. Reed, L. \& Muench, H. A simple method of estimating fifty per cent endpoints. Am. J. Epidemiol. 27, 493-497 (1938).

32. Krammer, F. \& Palese, P. Influenza virus hemagglutinin stalk-based antibodies and vaccines. Curr. Opin. Virol. 3, 521-530 (2013).

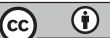

Open Access This article is licensed under a Creative Commons Attribution 4.0 International License, which permits use, sharing, adaptation, distribution and reproduction in any medium or format, as long as you give appropriate credit to the original author(s) and the source, provide a link to the Creative Commons license, and indicate if changes were made. The images or other third party material in this article are included in the article's Creative Commons license, unless indicated otherwise in a credit line to the material. If material is not included in the article's Creative Commons license and your intended use is not permitted by statutory regulation or exceeds the permitted use, you will need to obtain permission directly from the copyright holder. To view a copy of this license, visit http://creativecommons. org/licenses/by/4.0/.

(c) The Author(s) 2017 